\title{
Medial Wall Orbital Reconstruction using Unsintered Hydroxyapatite Particles/Poly L-Lactide Composite Implants
}

\author{
Hojin Park, \\ Hyon-Surk Kim, \\ Byung-Il Lee \\ Department of Plastic Surgery, \\ Korea University Anam Hospital, \\ Korea University College of Medicine, \\ Seoul, Korea
}

No potential conflict of interest relevant to this article was reported.

\begin{abstract}
Background: Poly-L-lactide materials combined with hydroxyapatite (u-HA /PLLA) have been developed to overcome the drawbacks of absorbable materials, such as radiolucency and comparably less implant strength. This study was designed to evaluate the usefulness of u-HA/PLLA material in the repair of orbital medial wall defects.

Methods: This study included 10 patients with pure medial wall blow-out fractures. The plain radiographs were taken preoperatively, immediately after, and 2 months after surgery. The computed tomography scans were performed preoperatively and 2 months after surgery. Patients were evaluated for ease of manipulation, implant immobility, rigidity and complications with radiologic studies.

Results: None of the patients had postoperative complications, such as infection or enophthalmos. The u-HA/PLLA implants had adequate rigidity, durability, and stable position on follow-up radiographic studies. On average, implants were thawed 3.4 times and required 14 minutes of handling time.

Conclusion: The u-HA/PLLA implants are safe and reliable for reconstruction of orbital medial wall in terms of rigidity, immobility, radiopacity, and cost-effectiveness. These thin yet rigid implants can be useful where wide periosteal dissection is difficult due to defect location or size. Since the u-HA/PLLA material is difficult to manipulate, these implants are not suitable for use in complex 3-dimensional defects.
\end{abstract}

Keywords: Orbital fractures / Orbital implants / Absorbable implants

\section{INTRODUCTION}

The ideal implant for blow-out fracture reconstruction should have good biocompatibility, strength to contain orbital contents, sterilizability, and plasticity to shape the implant $[1,2]$. The implant would be radiopaque, to enable radiographic evaluation after surgery, but should not produce artifacts. Graft sources range from autogenous tissues, such as bone or cartilage, to alloplastic materials, with the latter currently considered the workhorse materials.

\footnotetext{
Correspondence: Byung-II Lee

Department of Plastic Surgery, Korea University Anam Hospital, Korea University College of Medicine, 73 Inchon-ro, Seongbuk-gu, Seoul 02841, Korea

E-mail: guro@korea.ac.kr

Received June 23, 2015 / Revised August 20, 2015 / Accepted August 23, 2015
}

Polymer used widely as alloplastic materials can be divided into absorbable and non-absorbable types. Absorbable implants are made from poly-L-lactide (PLLA), poly-L/DL-lactide (P(L/DL) LA) 70/30, polydioxanone, or PLLA/polyglycolic acid (PGA), all of which have enough biomechanical resistance to reconstruct the orbital wall. However, these polymers are not radiopaque, and implants made from these polymers cannot be evaluated by radiographic studies after implantation. Some absorbable materials do not remain durable until the bone defect heals [3]. Thus, the use of absorbable implants, especially for large defects of the orbital wall, has been limited. Non-absorbable types are usually made from porous polyethylene, with or without titanium reinforcement. These implants are easy to manipulate and at the same time 
are ridig. The downside is that they are expensive [4].

Currently, PLLA materials have been combined with hydroxyapatite to overcome the drawbacks of absorbable implants, such as radiolucency, limited durability, and low strength. Despite these advantages, the unsintered hydroxyapatite (u-HA)/PLLA composite device retains the absorbable property. It consists of composite $\mathrm{u}-\mathrm{HA}$, carbonate ion, fine particles, and PLLA. The devices are bioactive and biodegradable, and u-HA/PLLA composites have the potential for total replacement of bone $[5,6]$.

In this study, we aimed to investigate the usefulness of u-HA/ PLLA in medial orbital wall reconstruction.

\section{METHODS}

A single-institutional prospective study was performed for all patients with medial wlal blowout fractures between October 2014 to February 2015. All of the patients had pure medial orbital wall fractures; those who had non-pure orbital wall fractures, or combined medial and orbital floor fractures, were excluded from the study. Surgical indication was made for expected future enophthalmos, defects greater than $2.5 \mathrm{~cm}^{2}$, extraocular movement impairment, and/or diplopia. All of the patients underwent reconstruction of the orbital wall using the u-HA/PLLA implants (Osteotrans MX ${ }^{\circledR}$, Takiron Co., Osaka, Japan). The operations were performed by the same surgeon in all cases.

Patients routinely underwent 3-dimensional computed tomography (CT) imaging and plain radiographs. The plain radiographs were taken preoperatively, immediately after, and at 2 months after surgery. The CT scans were performed preoperatively and at 2 months after surgery. On preoperative CT images, the height and depth of medial orbital wall defects were measured using coronal and axial views. The defect size was estimated by multiplying the depth by the height, with the assumption that the defect was rhomboid in shape.

\section{Post-implantation immobility or migration}

Postoperative Waters' view radiographs were used to evalaute post-implantation immobility. The angle between the implanta- tion line and midline was defined as the implant angle. The implant line extended from the most superior to most inferior points of the implant. The line connecting the nasion and anterior nasal spine was defined as midline (Fig. 1). Postoperative CT (2 months after operation) was also used to evaluate any extrusion of the implant. An implant was considered to be extruded if the anterior border of the implant extended beyond the orbital rim.

\section{Implant rigidity and durability}

Implant rigidity was evaluated via Waters' view taken immediately and at 2 months after the operation. On the Waters' view, the midpoint of implant was regarded as the center of the orbital wall defect. The distance between midline and implant midpoint was measured. Any change in this distance was used as a proxy measure of insufficient rigidity (Fig. 2).

\section{Handling time and number of thawing and bending}

The implant handling time was defined as the time spent in trimming, molding, and insertion of the implant into the orbital de-

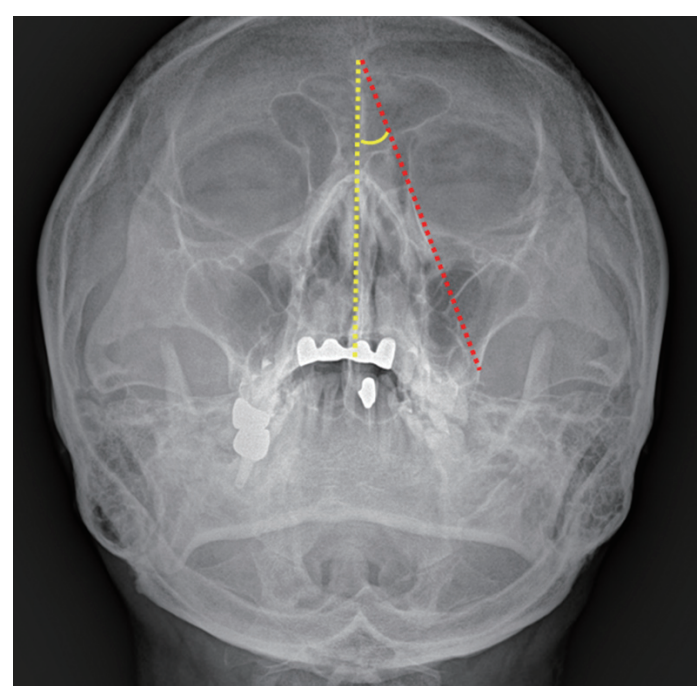

Fig. 1. Implant angle. The midline was drawn by connecting the nasion and anterior nasal spine (yellow line). The implant line extended from the most superior point to the most inferior point of the implant (red line). The angle between these two lines were used to evaluate implant ridigidity. 


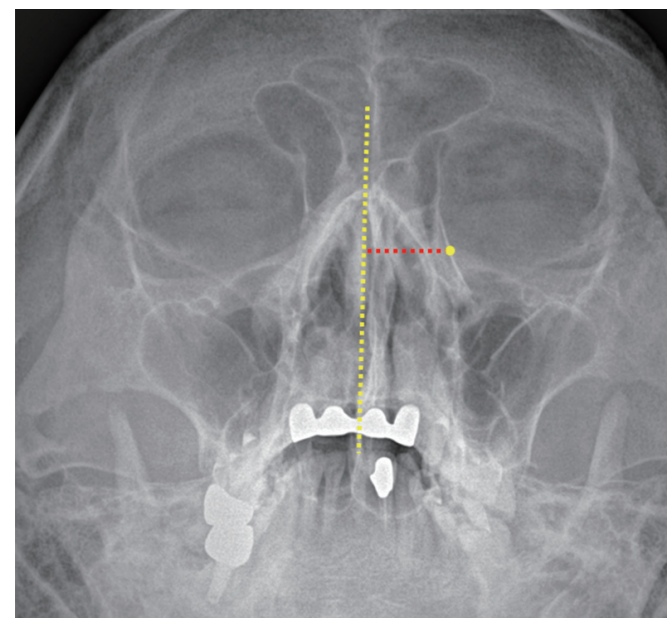

Fig. 2. Implant rigidity measurement. The distance (red line) was measured between the midline (yellow line) and the midpoint of the implant. Line connecting the nasion and anterior nasal spine was the midline (yellow line).

fect. Also, we counted the number of thawing and bending of the implant. The handling time and number of thawing and bending were used to interpret the ease of implant use.

\section{Operative technique}

All surgical procedures were performed under general anesthesia. A transcaruncular approach was used to access the orbital medial wall. Once the fracture site was exposed, the defect dimensions was measured using a paper ruler. We made a template of the orbital defect with a sterile piece of paper and used this template to estimated the size and shape of the defect. The implant was trimmed and molded to fit the anatomic shape of orbital wall. To increase pliability of the u-HA/PLLA material, the implant was submerged in $65^{\circ} \mathrm{C}$ water for 30 -to- 60 seconds. Once molded to shape, the implant was placed over the defect under the periosteum, and was fixated at points $0.5 \mathrm{~cm}$ posterior to the posterior lacrimal crest. We did not fix the implant with screws. The periosteum was repaired with one or two point sutures using 6-0 Vicryl. The skin incisionw as also closed with absorbable sutures.

\section{Statistical analysis}

All results are expressed as mean and standard deviation. Data analyses were performed using SAS ver. 9.2 statistical software program (SAS Institute, Inc., Cary, NC, USA). Statistical tests included the paired $t$-test, which was applied to assess changes of implant position in rigidity analysis and the implant angle. P-values less than 0.05 were considered statistically significant.

\section{RESULTS}

The prospective study included 10 patients ( 9 male and 1 female) who underwent reconstruction of blowout fractures with u-HA/ PLLA. The mean orbital defect size was $2.1 \mathrm{~cm}^{2}$ (Table 1). None of the patients experienced complications related to surgery, such as infection or enophthalmos throughout the follow-up period. The u-HA/PLLA implants remained radiopaque and could be identified in all patients on follow-up radiologic study (Fig. 3).

\section{Post-implantation immobility or migration}

The mean implant angles were $12.3^{\circ}$ immediately after surgery and $12.4^{\circ}$ at the 2 -month follow-up. This was not a statistically sig-

Table 1. Patient demographics

\begin{tabular}{lc} 
Characteristic & u-HAPLLA \\
\hline Number & 10 \\
\hline Age $(y r)$ & $37.4 \pm 14.4$ \\
\hline Sex (male:female) & $9: 1$ \\
\hline Defect size $\left(\mathrm{cm}^{2}\right)$ & $2.1 \pm 0.7$ \\
\hline
\end{tabular}

u-HA, unsintered hydroxyapatite; PPLA, poly-L-lactide.
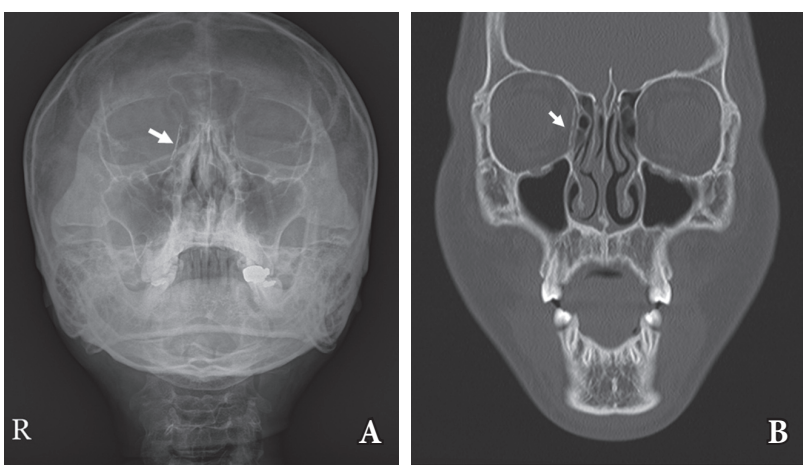

Fig. 3. Radiopacity of u-HA/PLLA. The u-HA/PLLA is radiopaque on plain radiographs (A) and computed tomography images (B). White arrows indicate the implant in a patient with a right medial wall blowout fracture. u-HA, unsintered hydroxyapatite; PPLA, poly-L-lactide. 
nificant difference $(p=0.38)$ (Table 2). None of the implants had undergone extrusion on follow-up CT evaluations.

\section{Implant rigidity and durability}

The distance between the midpoint of implant and midline was $1.80 \mathrm{~cm}$ immediately after surgery and $1.78 \mathrm{~cm}$ at 2-month followup. On paired $t$-test, this was not a statistically significant difference $(p=0.39)$ (Table 2).

\section{Handling time and number of thawing and bending}

The mean time spent handling the u-HA/PLLA implant was 14 minutes. On average, the implants were thawed 3.4 times (range, 2-5 times).

\section{DISCUSSION}

The u-HA/PLLA material is made from combining the non-calcified and unsintered HA and the PLLA. This composite material is produced by a unique compression molding process. Its elasticity is similar to that of the cortical bone, and the material retains high strength through the time required for bone healing. It also has optimal degradation and resorption behavior, and is osteoconductive [5]. Hydroxyapatite, a component of this device, is not resorbable. However, the $\mathrm{u}-\mathrm{HA}$ crystal is small enough to trigger phagocytosis, with incorporation into the PLLA matrix and, as such, exhibits effective bioactivity [7]. However, the mechanical properties have not been reported for u-HA/PLLA.

Implant immobility is major concern after surgical treatment of blow-out fractures. Alloplastic materials have been associated with implant extrusion, although the actual rates are quite low $[8,9]$. Implant migration or extrusion is mainly due to inadequate

Table 2. The change of implant angle and mid-point midline distance

\begin{tabular}{lccc} 
Variable & Immediate & 2 months & $p$-value ${ }^{\mathrm{a}}$ \\
\hline Implant angle $\left({ }^{\circ}\right)$ & $12.3 \pm 10.6$ & $12.4 \pm 10.7$ & 0.38 \\
Mid-point midline distance $(\mathrm{cm})$ & $1.80 \pm 0.28$ & $1.78 \pm 0.24$ & 0.39 \\
\hline
\end{tabular}

subperiosteal dissection for its insertion. This is especially the case when a defect is relatively large or close to the apex of the orbit. Screw fixation is sometimes helpful in preventing implant migration, but the medial orbital wall is usually too thin to provide the anchoring support for screws.

Although none of the u-HA/PLLA implants had migrated, the authors have observed porous polyethylene/titanium implants undergoing migration due to insufficient subperiosteal pocket. The porous polyethylene/titanium implants are usually $0.8 \mathrm{~mm}$ thick, while th u-HA/PLLA implants are usually $0.5 \mathrm{~mm}$ thick. Although the absolute difference in thickness is just $0.3 \mathrm{~mm}$, it tranlates into the porous polyethylene/titanium having 1.6 times the thickness of the u-HA/PLLA material. This increases the chance of extrusion for porous polyetheylene/titanium mesh, if the subperiosteal pocket cannot be created within the defect margin. The $\mathrm{u}-\mathrm{HA} / \mathrm{PLLA}$ is thinner than most absorbable materials. Polycaprolactone and P(L/DL)LA 70/30 implants are $0.75 \mathrm{~mm}$ and $0.6 \mathrm{~mm}$ thick, respectively. The LPLA/PGA implant is 1.5 $\mathrm{mm}$ thick. Because of this, the u-HA/PLLA implant appears to be useful when wide dissection is difficult because of a defect is too large or too close to the apex of the orbit. Orbital wall implants should have enough rigidity to contain the orbital contents without undergoing mechanical deformation. This rigidity is determined by the ratio of components making the up implant material. The composite u-HA/PLLA material has an initial bending strength of up to $270 \mathrm{Mpa}$ (27.0 MPa or $270 \mathrm{MPa}$ ) [10].

Ideally, absorbable materials should provide appropriate strength while degrading at a predictable rate. The u-HA/PLLA material is known to maintain a strength equal to that of human cortical bone for 25 weeks after implantation [6]. Thus, it is expected to maintain structural strength while the bony defect heals. The material also has osteoconductivity, which allows osteoblasts to attach to the implant, and the implant can then be replaced by bone.

To measure the rigidity of implant, we evaluated Waters' view radiographs taken immediately and 2 months after the operation. This distance did not change from immediate postop period to the 2-month follow up exam. In our study, u-HA/PLLA appears to maintain enough rigidity for the 2-months follow up period. 
However, additional studies are needed to understand rigidity and durability of the implant over the full course of bone remodeling. In a future study, we expect to confirm the durability, strength, and osteoconductivity of u-HA/PLLA.

Baek et al. [11] compared the long term results of titanium mesh plate and absorbable implant, in which study implant rigidity was evaluated for postoperative complications including diplopia and enophthalmos. You et al. [2] also studied the long term results after absorbable implant reconstruction by measuring the difference in herniated soft tissue between preoperative and postoperative observations. The authors believed that herniated volume difference or postoperative enophthalmos was more dependent on surgical skills than the natural rigidity or durability of an implant. Because of this, we had measured implants position or shape change on radiologic studies to analyze rigidity of u-HA/PLLA.

Unlike most absorbable imlpants, the u-HA/PLLA material is radiopaque on plain radiographs and CT images because the unsintered hydroxyappetite has radioabsorptive density similar to that of bone. Thus, the implant location can be followed for migration and extrusion on plain X-ray or CT scans.

The major drawback of the u-HA/PLLA material is shared across all absorbable materials-the difficulty in manipulation. To form a curve that conforms to the anatomic shape of medial wall, we needed to warm up and mold the u-HA/PLLA implant through several cycles during each operation. Moreover, the warmed u-HA/ PLLA implant becomes solidified more rapidly than other absorbable materials do. Thus, u-HA/PLLA implants required an increased number of thawing and bending and subsequent increase in handling time. Unlike u-HA/PLLA, non-absorbable polymers like porous polyethylene/titanium can be adjusted after insertion, due to the malleability afforded by the titanium component.

For cost-effectiveness, u-HA/PLLA is superior to other implants. The most commonly used size of $\mathrm{u}-\mathrm{HA} / \mathrm{PLLA}$ products costs about $\$ 800$ USD, which compared favorable to the cost of porous polyethylene $(\$ 1,300)$ and polyethylene/titanium meshes $(\$ 1,700)$. The cost of other absorbable implants ranges from 850 USD to 1,000 USD. Currently, the u-HA/PLLA implant is more cost-effective than porous polyethylene/titanium and other absorbable implants.
Limitations of this study include the small sample size, a relatively short follow-up, and the absence of CT images taken immediately after surgery. Long-term follow up of these patients is necessary to evaluate bony change occuring within the implant. Although no patients in our study demonstrated signs of inflammation, long-term follow-up is needed to evaluate foreign body reactions. Plain radiographs were used to evaluate immobility and rigidity in our study, but these would be more accurately evaluated on CT imaging. In the future, CT images taken immediately and 2 months after surgery are needed to more accurately compare the shape and position of the implant. At the moment, immediate postoperative CT examination is not covered under the national health insurance payout schedule in South Korea and such a study would be cost-prohibited for the time being.

In conclusion, the u-HA/PLLA implants are safe and reliable for reconstruction of the medial wall of the orbit, in terms of rigidity, immobility, radiopacity, and cost-effectiveness. Moreover, these implants can be useful when the enough subperiosteal pocket cannot be created from the defect margin, either because the defect is too close to the orbital apex or becasue the defect is large. Similar to other absorbable implants, u-HA/PLLA implants are difficult to manipulate and mold, and are inconvenient for reconstruction of a complex 3-dimensional defect.

\section{REFERENCES}

1. Potter JK, Ellis E. Biomaterials for reconstruction of the internal orbit. J Oral Maxillofac Surg 2004;62:1280-97.

2. You JP, Kim DW, Jeon BJ, Jeong SH, Han SK, Dhong ES, et al. Twoyear follow-up on the use of absorbable mesh plates in the treatment of medial orbital wall fractures. Arch Plast Surg 2013;40:728-34

3. Gunarajah DR, Samman N. Biomaterials for repair of orbital floor blowout fractures: a systematic review. J Oral Maxillofac Surg 2013;71: 550-70.

4. Han DH, Chi M. Comparison of the outcomes of blowout fracture repair according to the orbital implant. J Craniofac Surg 2011;22:1422-5.

5. Hayashi M, Muramatsu H, Sato M, Tomizuka Y, Inoue M, Yoshimoto S. Surgical treatment of facial fracture by using unsintered hydroxyapatite particles/poly l-lactide composite device (OSTEOTRANS MX((R))): a clinical study on 17 cases. JCraniomaxillofac Surg 2013;41:783-8.

6. Yasunaga T, Matsusue Y, Furukawa T, Shikinami Y, Okuno M, Nakamura T. Bonding behavior of ultrahigh strength unsintered hydroxyapatite particles/poly(L-lactide) composites to surface of tibial cortex 
in rabbits. J Biomed Mater Res 1999;47:412-9.

7. Shikinami Y, Matsusue Y, Nakamura T. The complete process of bioresorption and bone replacement using devices made of forged composites of raw hydroxyapatite particles/poly l-lactide (F-u-HA/ PLLA). Biomaterials 2005;26:5542-51.

8. Miyasaka M, Tanaka R, Hanai U, Yamazaki A, Iida M, Akamatsu T. A case of chronic infection 28 years after silicone orbital implant. Tokai J Exp Clin Med 2008;33:35-8.

9. Morrison AD, Sanderson RC, Moos KF. The use of silastic as an orbit- al implant for reconstruction of orbital wall defects: review of 311 cases treated over 20 years. J Oral Maxillofac Surg 1995;53:412-7.

10. Shikinami Y, Okuno M. Bioresorbable devices made of forged composites of hydroxyapatite (HA) particles and poly-L-lactide (PLLA): Part I. Basic characteristics. Biomaterials 1999;20:859-77.

11. Baek WI, Kim HK, Kim WS, Bae TH. Comparison of absorbable mesh plate versus titanium-dynamic mesh plate in reconstruction of blow-out fracture: an analysis of long-term outcomes. Arch Plast Surg 2014;41:355-61. 